Kyoto University,

Graduate School of Economics

Research Project Center Discussion Paper Series

\title{
Options on Multiple Assets in a Mean-Reverting Model
}

Masahiko Egami and Tadao Oryu

Discussion Paper No. E-10-005

Research Project Center

Graduate School of Economics

Kyoto University

Yoshida-Hommachi, Sakyo-ku

Kyoto City, 606-8501, Japan

July 2010 


\title{
OPTIONS ON MULTIPLE ASSETS IN A MEAN-REVERTING MODEL
}

\author{
MASAHIKO EGAMI AND TADAO ORYU
}

\begin{abstract}
We solve two optimal stopping problems whose payoff functions are the maximum and the minimum of two state variables driven by the Ornstein-Uhlenbeck processes. We consider a class of problems where we obtain analytical solutions. Furthermore, by making use of the analytical results we study some properties of exercise regions including convexity, symmetry, and continuity.
\end{abstract}

Key words: American options on multiple assets, Optimal stopping, Mean-reverting model. JEL Classification: C11 G13

Mathematics Subject Classification (2000) : Primary 91B28 Secondary 60G45, 62L15

\section{INTRODUCTION}

There are various derivatives traded today in the financial markets. Many of those have payoffs based on the only one underlying asset, but some derivatives relate to two or more assets; for example, the one pays difference of prices of two assets, or the one brings the asset whose price is higher than the other. There are no general methods to solve the pricing and hedging problems for American-type derivatives related to multiple assets. These problems lead to optimal stopping problems in higher dimensions. Broadie and Detemple [2] characterize the option exercise regions and provide valuation formulae for several kinds of payoff functions. Under the Black-Scholes setup, for example, they consider American call options on the maximum of two stocks with payoff function $f\left(x_{1}, x_{2}\right)=\left(\max \left(x_{1}, x_{2}\right)-K\right)^{+}$and showed, among other things, that it is not optimal to exercise when the prices of the underling assets are equal and that each of the two subregions of early exercise regions is convex (page 247 in [2]). Following this, Villeneuve [11] extended [2] by analyzing additional payoff functions and studied the notion of critical surface for which one can extend some results in the one-dimensional case. More recently, Detemple et al. [7] show, for call options on the minimum of two dividend-paying assets, that the optimal exercise boundary consists of three components, two continuous curves and one component along the diagonal with empty interior (page 955, Figure 1). Guillaume [9] further extends in the direction that more assets are included in the payoff with knock-in and knock-out provisions and also that the pricing of these contracts is made in a multivariate jump-diffusion framework allowing for a stochastic two-factor term structure of interest rates. Also see Detemple [6] and the references therein.

Motivated by the foregoing studies, in this article, we shall find exercise regions for derivatives that pay the higher value of two assets following the Ornstein-Uhlenbeck processes. One can think of derivatives

This research is in part supported by Grant-in-Aid for Scientific Research (C) No. 20530340, Japan Society for the Promotion of Science. 
associated with interest rates in financial engineering or certain state variables whose dynamics are appropriately modeled by mean-reverting processes in the theory of investment under uncertainty. For the latter case, see for example Cadenillas et al. [3] and Bayraktar and Egami [1]. To our knowledge, this is a first attempt to handle options on multiple assets driven by mean-reverting diffusion processes. Since it is of practical importance to identify the shape of exercise regions for better understanding of options on multiple assets, this direction of research is quite meaningful. While the papers in the literature use numerical methods to identify exercising boundaries ${ }^{1}$, we shall solve the problem analytically in an infinite horizon model. The benefits of obtaining analytical solutions include the following: we believe that the results here can serve as a benchmark against which one can compare more general cases (that require numerical solutions), shedding lights to the theoretical aspect of this problem. Moreover, we can make a rigorous mathematical analysis on the shape of exercise boundaries by taking advantage of explicit formulae. For this purpose, we introduce "the difference of the two processes" as a new process and represent the payoff solely by this new process, so that we reduce the dimension of the problem to one, an effective technique, e.g. Davis and Norman [4]. Indeed, we have a family of optimal stopping problems in one-dimension parameterized by a function of the initial values of the two OU processes. After solving the family of problems, we then come back to the original problem and identify the exact shape of the exercise boundary that turns out to consist of several segments. The continuity of these segments are also rigorously proved. Moreover, we perform some comparative statics on the parameters to better grasp qualitative aspects of the value of the option.

This paper is constructed as follows. We solve the optimal stopping problem on the maximum of the two assets in Section 2 and present a numerical example, discussing the properties of the exercise regions in Section 3. In Section 4, we solve the problem on the minimum of the two with a numerical example as well. We find that the exercise region for this option is a subset of the exercise region for the maximum option. Section 5 is an appendix which explains the outline of the solution method (for optimal stopping) based on Dynkin [8] and Dayanik and Karatzas [5].

\section{Maximum Option}

2.1. The Model Setup. Let the stochastic processes $X^{i}=\left\{X_{t}^{i} ; t \geq 0\right\}(i=1,2)$ be the prices of the two assets, defined on probability space $(\Omega, \mathcal{F}, \mathbb{P})$, where $\Omega$ is the set of all possible realization of the stochastic economy, and $\mathbb{P}$ is a risk-neutral measure defined on $\mathcal{F}$. We denote by $\mathbb{F}=\left\{\mathcal{F}_{t}\right\}$ the filtration with respect to which $X^{1}$ and $X^{2}$ are adapted and with the usual conditions being satisfied. $X^{1}$ and $X^{2}$ satisfy the stochastic differential equations, with $X_{0}^{1}=x_{1}$ and $X_{0}^{2}=x_{2}$,

$$
\begin{aligned}
\mathrm{d} X_{t}^{i} & =-\alpha X_{t}^{i} \mathrm{~d} t+\sigma_{i} \mathrm{~d} B_{t}^{i} \quad(i=1,2), \\
\mathrm{d}\left[B^{1}, B^{2}\right]_{t} & =\rho \mathrm{d} t,
\end{aligned}
$$

where $\alpha$ and $\sigma_{i}$ are positive constants, and $B^{1}$ and $B^{2}$ are standard Brownian Motions on $(\Omega, \mathcal{F}, \mathbb{P})$. We set the common parameter $\alpha$ here since the reduction of the dimension seems impossible if they are

\footnotetext{
${ }^{1}$ There are no analytical solutions found so far for multi-dimension optimal stopping problems or for finite-time horizon problems.
} 
distinct. For this case, we have to resort to numerical solutions. The Ornstein-Uhlenbeck processes is frequently used for representing the dynamics of the assets that have the tendency of mean-reversion. Hence one can understand that our problem here is associated with interest rates or prices of cyclical products. Note that we set the mean-reverting level zero here. Since a simple translation can handle the non-zero mean level easily, we solve our problem based on the dynamics (2.1). It is well known that $X^{1}$ and $X^{2}$ are represented of the closed form,

$$
X_{t}^{i}=e^{-\alpha t} x_{i}+\sigma_{i} \int_{0}^{t} e^{-\alpha(t-s)} \mathrm{d} B_{s}^{i} .
$$

Here the same $\alpha$ is set for both $X^{i}$. It is clearly better that $\alpha_{1}$ and $\alpha_{2}$ are used in the equations of $X^{1}$ and $X^{2}$ respectively, but the condition of the same $\alpha$ is needed for the method of dimensional reduction offered in this paper. The function is $\Phi\left(x_{1}, x_{2}\right)=x^{1} \vee x^{2}$, and the value function $V: \mathbb{R} \times \mathbb{R} \mapsto \mathbb{R}$ is defined by

$$
V\left(x_{1}, x_{2}\right)=\sup _{\tau \in \mathcal{S}} \mathbb{E}_{x_{1}, x_{2}}\left[e^{-r \tau} \Phi\left(X_{\tau}^{1}, X_{\tau}^{2}\right)\right],
$$

where $r$ is constant discount rate $(r>0)$, and $\mathcal{S}$ is the set of all possible $\mathbb{F}$-stopping times.

2.2. Reduction of the Dimension. To reduce the dimension of the problem, we define the new process $X^{e}=X^{1}-X^{2}$, then $X^{e}$ satisfies the stochastic differential equation

$$
d X_{t}^{e}=-\alpha X_{t}^{e} d t+\sigma_{e} d B_{t}^{e}
$$

where

$$
\sigma_{e}=\sqrt{\sigma_{1}^{2}-2 \rho \sigma_{1} \sigma_{2}+\sigma_{2}^{2}}, \quad \text { and } \quad B_{t}^{e}=\frac{1}{\sigma_{e}}\left(\sigma_{1} B_{t}^{1}-\sigma_{2} B_{t}^{2}\right)
$$

$B^{e}$ is a standard Brownian motion on $(\Omega, \mathcal{F}, \mathbb{P})$ because it is a continuous martingale that starts at 0 and

$$
\begin{aligned}
\mathrm{d}\left[B^{e}, B^{e}\right]_{t} & =\frac{1}{\sigma_{e}^{2}}\left(\sigma_{1}^{2} \mathrm{~d}\left[B^{1}, B^{1}\right]_{t}-2 \sigma_{1} \sigma_{2} \mathrm{~d}\left[B^{1}, B^{2}\right]_{t}+\sigma_{2}^{2} \mathrm{~d}\left[B^{2}, B^{2}\right]_{t}\right) \\
& =\frac{1}{\sigma_{e}^{2}}\left(\sigma_{1}^{2}-2 \rho \sigma_{1} \sigma_{2}+\sigma_{2}^{2}\right) \mathrm{d} t \\
& =\mathrm{d} t
\end{aligned}
$$

Since $\alpha, r>0$ and $\tau \geq 0$ a.s.,

$$
\begin{aligned}
\mathbb{E}_{x_{1}, x_{2}}\left[\left(e^{-r \tau} \int_{0}^{\tau} e^{-\alpha(\tau-s)} \mathrm{d} B_{t}^{i}\right)^{2}\right] & =\mathbb{E}_{x_{1}, x_{2}}\left[e^{-2 r \tau} \int_{0}^{\tau} e^{-2 \alpha(\tau-s)} \mathrm{d} s\right] \\
& =\mathbb{E}_{x_{1}, x_{2}}\left[e^{-2(\alpha+r) \tau} \int_{0}^{\tau} e^{2 \alpha s} \mathrm{~d} s\right] \\
& =\frac{1}{2 \alpha} \mathbb{E}_{x_{1}, x_{2}}\left[e^{-r \tau}\left(1-e^{-2 \alpha \tau}\right)\right] \leq \frac{1}{2 \alpha}<\infty
\end{aligned}
$$

so for any $\tau \in \mathcal{S}, e^{-r \tau} \int_{0}^{\tau} e^{-\alpha(\tau-s)} \mathrm{d} B_{t}^{i}$ is integrable and then

$$
\mathbb{E}_{x_{1}, x_{2}}\left[e^{-r \tau} \int_{0}^{\tau} e^{-\alpha(\tau-s)} \mathrm{d} B_{t}^{i}\right]=0
$$


Therefore the following equations are satisfied;

$$
\begin{aligned}
\mathbb{E}_{x_{1}, x_{2}}\left[e^{-r \tau}\left(X_{\tau}^{2}\right)\right] & =\mathbb{E}_{x_{1}, x_{2}}\left[e^{-(\alpha+r) \tau} x_{2}+\sigma_{2} e^{-r \tau} \int_{0}^{\tau} e^{-\alpha(\tau-s)} \mathrm{d} B_{s}^{2}\right] \\
& =x_{2} \mathbb{E}_{x_{1}, x_{2}}\left[e^{-(\alpha+r) \tau}\right], \\
\mathbb{E}_{x_{1}, x_{2}}\left[e^{-r \tau}\left(X_{\tau}^{e}\right)\right] & =\mathbb{E}_{x_{1}, x_{2}}\left[e^{-(\alpha+r) \tau}\left(x_{1}-x_{2}\right)+\sigma_{e} e^{-r \tau} \int_{0}^{\tau} e^{-\alpha(\tau-s)} \mathrm{d} B_{s}^{e}\right] \\
& =\left(x_{1}-x_{2}\right) \mathbb{E}_{x_{1}, x_{2}}\left[e^{-(\alpha+r) \tau}\right] .
\end{aligned}
$$

By these equations, we can reduce the dimension, that is, we can represent the value function

$$
\begin{aligned}
V\left(x_{1}, x_{2}\right) & =\sup _{\tau \in \mathcal{S}} \mathbb{E}_{x_{1}, x_{2}}\left[e^{-r \tau}\left(X_{\tau}^{1} \vee X_{\tau}^{2}\right)\right] \\
& =\sup _{\tau \in \mathcal{S}} \mathbb{E}_{x_{1}, x_{2}}\left[e^{-r \tau}\left(X_{\tau}^{e}\right)^{+}+e^{-r \tau} X_{\tau}^{2}\right] \\
& =\sup _{\tau \in \mathcal{S}} \mathbb{E}_{x_{1}, x_{2}}\left[e^{-r \tau}\left(\left(X_{\tau}^{e}\right)^{+}+\frac{x_{2}}{x_{1}-x_{2}} X_{\tau}^{e}\right)\right] \\
& =\sup _{\tau \in \mathcal{S}} \mathbb{E}_{x_{1}, x_{2}}\left[e^{-r \tau} h\left(X_{\tau}^{e}\right)\right],
\end{aligned}
$$

where

$$
h(x)=\left\{\begin{array}{ll}
\frac{x_{1}}{x_{1}-x_{2}} x & (x \geq 0) \\
\frac{x_{2}}{x_{1}-x_{2}} x & (x<0)
\end{array} .\right.
$$

The original problem (2.2) is now represented by $X^{e}$ alone. For brevity, let

$$
p:=\frac{x_{1}}{x_{1}-x_{2}} \quad \text { and } \quad p-1:=\frac{x_{2}}{x_{1}-x_{2}}
$$

through the remainder of this article. We have thus reduced the original problem to a family of onedimensional optimal stopping problems parameterized by $p$. In fact, the property of the value function is quite different in the case $p \geq 1,0<p<1, p \leq 0$, which we shall examine below.

2.3. Solution. The outline of a general solution method for optimal stopping problem of one-dimensional diffusions is described in Appendix. In this section, we will use the method to solve our problem. The differential operator $\mathcal{A}$ for $X^{e}$ is defined by

$$
\mathcal{A} u(\cdot)=\frac{\sigma_{e}^{2}}{2} \frac{d^{2} u}{d x^{2}}(\cdot)-\alpha x \frac{d u}{d x}(\cdot) .
$$

The increasing and decreasing solution $\psi$ and $\varphi$ for the ordinary differential equation $(\mathcal{A}-r) u(x)=0$ (with $u \in C^{2}$ ) are known as

$$
\psi(x)=e^{\alpha x^{2} / 2} \mathcal{D}_{-r / \alpha}\left(\frac{-x \sqrt{2 \alpha}}{\sigma_{e}}\right) \quad \text { and } \quad \varphi(x)=e^{\alpha x^{2} / 2} \mathcal{D}_{-r / \alpha}\left(\frac{x \sqrt{2 \alpha}}{\sigma_{e}}\right),
$$

where $\mathcal{D}_{\nu}$ is the parabolic cylinder function denoted by

$$
\mathcal{D}_{\nu}(z)=2^{-\nu / 2} e^{-z^{2} / 4} \mathcal{H}_{\nu}(z / \sqrt{2}), z \in \mathbb{R}
$$


and $\mathcal{H}_{\nu}$ is the Hermite function of degree $\nu$ denoted by the integral representation

$$
\mathcal{H}_{\nu}(z)=\frac{1}{\Gamma(-\nu)} \int_{0}^{\infty} e^{-t^{2}-2 t z} t^{-\nu-1} d t, \operatorname{Re} \nu<0 .
$$

For these special functions, see Lebedev [10]. Let us define the transformation

$$
F(x):=\psi(x) / \varphi(x) \text { and } H(y):=(h / \varphi) \circ F^{-1}(y) .
$$

By the characterization of the value function as the nonnegative smallest majorant of $h / \varphi$ (see Proposition 5.2 in the appendix), we investigate the shape of the $H$ function in the transformed space.

Since $\lim _{x \rightarrow-\infty}\left(h^{+} / \varphi\right)(x)=0$, then $H(0)=0$, and it is clear from $F(0)=1$ and $h(0)=0$ that $H(1)=(h / \varphi)(0)=0$ (see Proposition 5.3). It is well-known that $H^{\prime \prime}(y)$ and $[(\mathcal{A}-r) h]\left(F^{-1}(y)\right)$ have the same sign. To calculate $[(\mathcal{A}-r) h](x)$ is easy;

$$
[(\mathcal{A}-r) h](x)= \begin{cases}-p(\alpha+r) x & (x \geq 0), \\ -(p-1)(\alpha+r) x & (x<0) .\end{cases}
$$

Because $F(x)$ is a monotone increasing function and $F(0)=1$, the sign of $H(y)$ and $H^{\prime \prime}(y)$ are determined as is shown in Table 1. In addition, it can be checked that these equations are satisfied;

$$
\lim _{y \rightarrow \infty} H(y)=\left\{\begin{array}{ll}
\infty & (p>0) \\
-\infty & (p \leq 0)
\end{array}, \quad \lim _{y \rightarrow \infty} H^{\prime}(y)=0 .\right.
$$

Now we have identified the complete description of $H(y)$, the next step is to find the smallest concave majorant of $H$. Recall however that the function $H$ depends on the parameter $p$.

2.3.1. Case (a) $\boldsymbol{p} \geq \mathbf{1}$. In this case, the function $H(y)$ is convex and negative on $[0,1)$, concave and positive on $(1,+\infty)$. It can be checked from $\lim _{y \rightarrow \infty} H^{\prime}(y)$ that there exists unique $z_{a}>1$, which is the unique solution of $y H^{\prime}(y)=H(y), y>0$. In our problem, the value of $z_{a}$ is independent of $p$.

Proposition 2.1. The solution $z_{a}$ of $y H^{\prime}(y)=H(y)$ is independent of $p$.

\begin{tabular}{|c|c|c|c|}
\hline \multicolumn{2}{|c|}{ cases } & the sign of $H(y)$ & the sign of $H^{\prime \prime}(y)$ \\
\hline (a) $p \geq 1$ & $y \geq 1$ & + & - \\
\cline { 2 - 4 } & $0<y<1$ & - & + \\
\hline \multirow{2}{*}{ (b) $0<p<1$} & $y \geq 1$ & + & - \\
\cline { 2 - 4 } & $0<y<1$ & + & - \\
\hline (c) $p \leq 0$ & $y \geq 1$ & - & + \\
\cline { 2 - 4 } & $0<y<1$ & + & - \\
\hline
\end{tabular}

TABLE 1. 
Proof. From the definition of $H(y)$ and the chain rule of derivative,

$$
\begin{aligned}
H(y) & =\left(\frac{h}{\varphi}\right) \circ F^{-1}(y) \\
& = \begin{cases}p\left(\frac{f}{\varphi}\right) \circ F^{-1}(y) \quad(y \geq 1), \\
(p-1)\left(\frac{f}{\varphi}\right) \circ F^{-1}(y) \quad(0 \leq y<1) .\end{cases} \\
y H^{\prime}(y) & =\frac{y}{F^{\prime}\left(F^{-1}(y)\right)}\left(\frac{h}{\varphi}\right)^{\prime} \circ F^{-1}(y) \\
& =y\left(\frac{h^{\prime} \varphi-h \varphi^{\prime}}{F^{\prime} \varphi^{2}}\right) \circ F^{-1}(y) \\
& = \begin{cases}p y\left(\frac{\varphi-f \varphi^{\prime}}{F^{\prime} \varphi^{2}}\right) \circ F^{-1}(y) & (y \geq 1), \\
(p-1) y\left(\frac{\varphi}{F^{\prime} \varphi^{2}}\right) \circ F^{-1}(y) & (0 \leq y<1) .\end{cases}
\end{aligned}
$$

where $f(x)=x$. Therefore $H(y)=y H^{\prime}(y)$ if and only if

$$
\left(\frac{f}{\varphi}\right) \circ F^{-1}(y)=y\left(\frac{\varphi-f \varphi^{\prime}}{F^{\prime} \varphi^{2}}\right) \circ F^{-1}(y) .
$$

This equation is independent of $p$.

The smallest nonnegative concave majorant $W_{a}(y)$ of $H(y)$ is thus

$$
W_{a}(y)= \begin{cases}\frac{y H\left(z_{a}\right)}{z_{a}} & \left(0<y \leq z_{a}\right) \\ H(y) & \left(y>z_{a}\right)\end{cases}
$$

If we define $x_{a}=F^{-1}\left(z_{a}\right)$, then $x_{a}>0$ and is also independent of $p$ by Proposition 2.1. The function $V_{a}(x)=\varphi(x) W_{a}(F(x))$ (see Proposition 5.3) is

$$
V_{a}(x)= \begin{cases}\frac{p x_{a}}{\psi\left(x_{a}\right)} \psi(x) & \left(x<x_{a}\right) \\ p x & \left(x \geq x_{a}\right)\end{cases}
$$

2.3.2. Case (b) $\mathbf{0}<\boldsymbol{p}<\mathbf{1}$. In this case, the function $H(y)$ is concave and positive on $[0,1)$ and on $(1, \infty)$. There exists unique pair $\left(z_{b, 1}, z_{b, 2}\right)$ with $z_{b, 1}<1<z_{b, 2}$ which is the solution of simultaneous equations:

$$
\left\{\begin{array}{l}
H^{\prime}\left(z_{b, 1}\right)=H^{\prime}\left(z_{b, 2}\right), \\
H\left(z_{b, 2}\right)-H\left(z_{b, 1}\right)=H^{\prime}\left(z_{b, 1}\right)\left(z_{b, 2}-z_{b, 1}\right) .
\end{array}\right.
$$

In contrast to $z_{a}$, the value of $\left(z_{b, 1}, z_{b, 2}\right)$ varies with $p$. The smallest nonnegative concave majorant $W_{b}(y)$ of $H(y)$ is

$$
W_{b}(y)= \begin{cases}H(y) & \left(0 \leq y<z_{b, 1}, z_{b, 2}<y\right), \\ \frac{H\left(z_{b, 2}\right)-H\left(z_{b, 1}\right)}{z_{b, 2}-z_{b, 1}}\left(y-z_{b, 1}\right)+H\left(z_{b, 1}\right) & \left(z_{b, 1} \leq y \leq z_{b, 2}\right) .\end{cases}
$$


If we define $z_{b, 1}=F\left(x_{b, 1}\right)$ and $z_{b, 2}=F\left(x_{b, 2}\right)$, then the function $V_{b}(x)=\varphi(x) W_{b}(F(x))$ is

$$
V_{b}(x)= \begin{cases}p x, & \left(x>x_{b, 2}\right), \\ (p-1) x & \left(x<x_{b, 1}\right), \\ \frac{p x_{b, 2} \varphi\left(x_{b, 1}\right)-(p-1) x_{b, 1} \varphi\left(x_{b, 1}\right)}{\varphi\left(x_{b, 1}\right) \psi\left(x_{b, 2}\right)-\varphi\left(x_{b, 2}\right) \psi\left(x_{b, 1}\right)} \varphi(x)-\frac{p x_{b, 2} \psi\left(x_{b, 1}\right)-(p-1) x_{b, 1} \psi\left(x_{b, 2}\right)}{\varphi\left(x_{b, 1}\right) \psi\left(x_{b, 2}\right)-\varphi\left(x_{b, 2}\right) \psi\left(x_{b, 1}\right)} \psi(x) & \left(x_{b, 1} \leq x \leq x_{b, 2}\right) .\end{cases}
$$

2.3.3. Case (c) $\boldsymbol{p} \leq \mathbf{0}$. In this case, the function $H(y)$ is concave and positive on $[0,1)$, and convex and negative on $(1, \infty)$. There exists unique $z_{c}<1$ which is the solution of $H^{\prime}(y)=0$. The value of $z_{c}$ is also independent of $p$ as $z_{a}$ is.

Proposition 2.2. The solution $z_{c}$ of $H^{\prime}(y)=0$ is independent of $p$.

Proof. It is clear from (2.4) that $H^{\prime}(y)=0$ if and only if

$$
\left(\frac{\varphi-f \varphi^{\prime}}{F^{\prime} \varphi^{2}}\right) \circ F^{-1}(y)=0
$$

This is independent of $p$.

The smallest nonnegative concave majorant $W_{c}(y)$ of $H(y)$ is

$$
W_{c}(y)= \begin{cases}H(y) & \left(0 \leq y<z_{c}\right), \\ H\left(z_{c}\right) & \left(y \geq z_{c}\right)\end{cases}
$$

If we define $z_{c}=F\left(x_{c}\right)$, then $x_{c}>0$ and is independent of $p$ by Proposition 2.2. The function $V_{c}(x)=$ $\varphi(x) W_{c}(F(x))$ is

$$
V_{c}(x)= \begin{cases}(p-1) x & \left(x \leq x_{c}\right), \\ \frac{(p-1) x_{c}}{\varphi\left(x_{c}\right)} \varphi(x) & \left(x>x_{c}\right) .\end{cases}
$$

The functions $V_{a}(x), V_{b}(x)$, and $V_{c}(x)$ are the value functions denoted by $x=x_{1}-x_{2}$ when $p$ is fixed. Since $p x_{2}=(p-1) x_{1}$ is satisfied from the definition of $p, p$ is constant on a line in $\left(x_{1}, x_{2}\right)$-plane through the origin. Therefore, $V_{a}(x), V_{b}(x)$, and $V_{c}(x)$ can be regarded as the cross sections of $V\left(x_{1}, x_{2}\right)$ cut by the plane $p x_{2}=(p-1) x_{1}$. Indeed, it is difficult to draw the graphs of $V\left(x_{1}, x_{2}\right)$ and $\mathcal{E}$, but the method mentioned above is enough to know the value of $V\left(x_{1}, x_{2}\right)$ and make decisions as to "exercise" or "continue".

\section{Properties of Exercise Regions}

In this section, we solve a numerical example by the method described in section 2 for the case $r=0.05, \alpha=1, \sigma_{1}=0.15, \sigma_{2}=0.125, \rho=0.75$, and then $\sigma_{e}=0.1$. Note that the solution in the previous section does not depend on these parameters. Hence the analysis here is applicable to a general situation of our problem. The values of $x_{a}=0.134435$ and $x_{c}=-0.134435$ are independent of $p$ as is shown in Proposition 2.1 and Proposition 2.2. Table 2 shows the values of $x_{b, 1}$ and $x_{b, 2}$ for five different $p$. The graphs of $W_{a}(y), W_{b}(y)$, and $W_{c}(y)$ are shown with $H_{a}(y), H_{b}(y)$, and $H_{c}(y)$ in Figure 1 , Figure 
2, and Figure 3, respectively. We also draw the graphs of $V_{a}(x), V_{b}(x)$, and $V_{c}(x)$ with $h(x)$ in each figure. The exercise region $\mathcal{E}$ is shown in Figure 4(i) as the upper and lower regions of the curves. Accordingly the continuation region is the area between the two curves. The two curves in the first quadrant (in Figure 4(i)) are straight lines, reflecting the fact that $z_{a}$ and $z_{c}$ are independent of $p$. The other parts of the curves (rather than the straight lines) in Figure 4(i) are drawn by the following steps (see also Figure 4(ii)):

step (i): Fix some $p \in(0,1)$.

step (ii): Draw the line $l_{p}: p x_{2}=(p-1) x_{1}$ on the $\left(x_{1}, x_{2}\right)$-plane.

step (iii): Calculate $x_{b, 1}$ and $x_{b, 2}$, and draw the lines $l_{1}: x_{1}-x_{2}=x_{b, 1}$ and $l_{2}: x_{1}-x_{2}=x_{b, 2}$. step (iv): Plot the intersection point of " $l_{p}$ and $l_{1}$ ", and that of " $l_{p}$ and $l_{2}$ ". (in fact, these points are $\left(p x_{b, 1},(p-1) x_{b, 1}\right)$ and $\left(p x_{b, 2},(p-1) x_{b, 2}\right)$ to be computed from easy calculations.) step (v): Return to step (i) with another $p \in(0,1)$.

\begin{tabular}{|c|c|c|c|c|c|}
\hline$p$ & 0.3 & 0.4 & 0.5 & 0.6 & 0.7 \\
\hline$x_{b, 1}$ & -0.134808 & -0.139114 & -0.150892 & -0.174689 & -0.225930 \\
\hline$x_{b, 2}$ & 0.225930 & 0.174689 & 0.150892 & 0.139114 & 0.1348098 \\
\hline
\end{tabular}

TABLE 2.

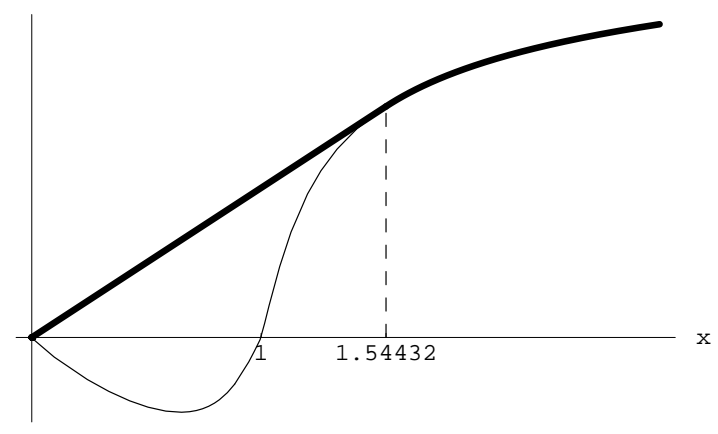

(i)

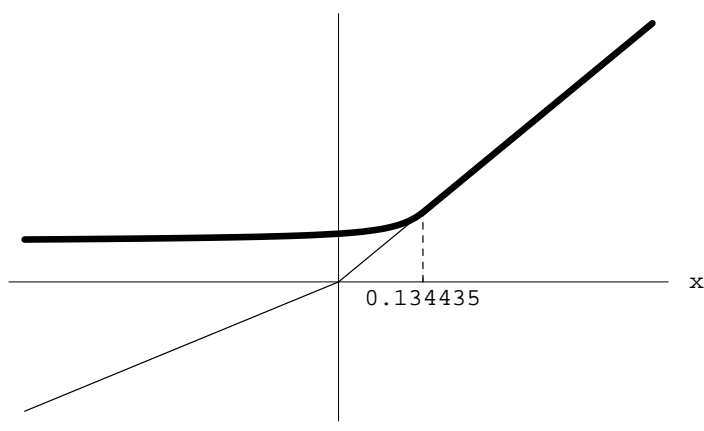

(ii)

Figure 1. $\mathbf{p}=\mathbf{2}$. The solid line in (i) is $H_{a}(y)$ and the thick line is $W_{a}(y)$. The solid line in (ii) is $h(x)$ and the thick line is $V_{a}(x) .1 .54432=z_{a}$, and $0.134435=x_{a}=F^{-1}\left(z_{a}\right)$.

3.1. Properties of Exercise Region. By viewing Figure 4, the exercise region, denoted by $\mathcal{E}$, is divided into two parts. In the $x_{1}-x_{2}$ plane, $x_{2}=E_{1}\left(x_{1}\right)$ and $x_{1}=E_{2}\left(x_{2}\right)$ are the two curves which are the boundaries between the continuation and exercise regions. That is, $\mathcal{E}=\mathcal{E}_{1} \cup \mathcal{E}_{2}$, where $\mathcal{E}_{1}:=\left\{\left(x_{1}, x_{2}\right) \in\right.$ $\left.\mathbb{R}^{2} ; x_{2} \geq E_{1}\left(x_{1}\right)\right\}$ and $\mathcal{E}_{2}:=\left\{\left(x_{1}, x_{2}\right) \in \mathbb{R}^{2} ; x_{1} \geq E_{2}\left(x_{2}\right)\right\}$. It seems that $\mathcal{E}_{1}, \mathcal{E}_{2}, E_{1}\left(x_{1}\right)$, and $E_{2}\left(x_{2}\right)$ have following properties:

(i) Convexity : If $\left(x_{1}, x_{2}\right) \in \mathcal{E}_{1}$ and $\left(x_{1}^{\prime}, x_{2}^{\prime}\right) \in \mathcal{E}_{1}$, then $\left(\lambda x_{1}+(1-\lambda) x_{1}^{\prime}, \lambda x_{2}+(1-\lambda) x_{2}^{\prime}\right) \in \mathcal{E}_{1}$ for all $\lambda \in[0,1]$ (and the same thing about $\mathcal{E}_{2}$ is true). 


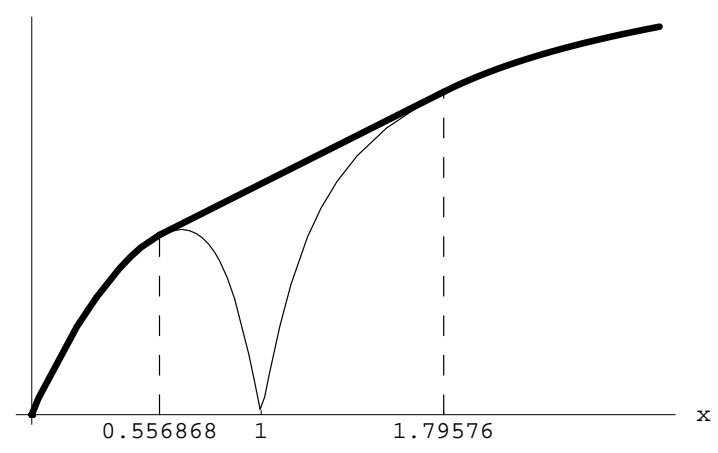

(i)

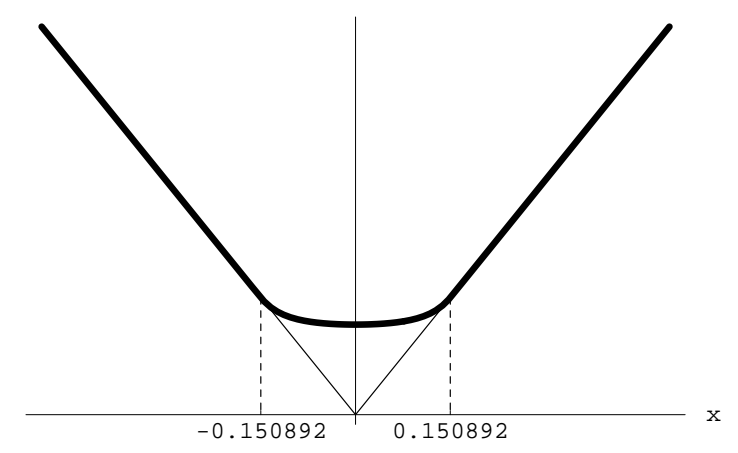

(ii)

Figure 2. $\mathbf{p}=\mathbf{0 . 5}$. The solid line in (i) is $H_{b}(y)$ and the thick line is $W_{b}(y)$. The solid line in (ii) is $h(x)$ and the thick line is $V_{b}(x) .0 .556868=z_{b, 1}, 1.79576=z_{b, 2}$, $-0.150892=x_{b, 1}=F^{-1}\left(z_{b, 1}\right)$, and $0.150892=x_{b, 2}=F^{-1}\left(z_{b, 2}\right)$.

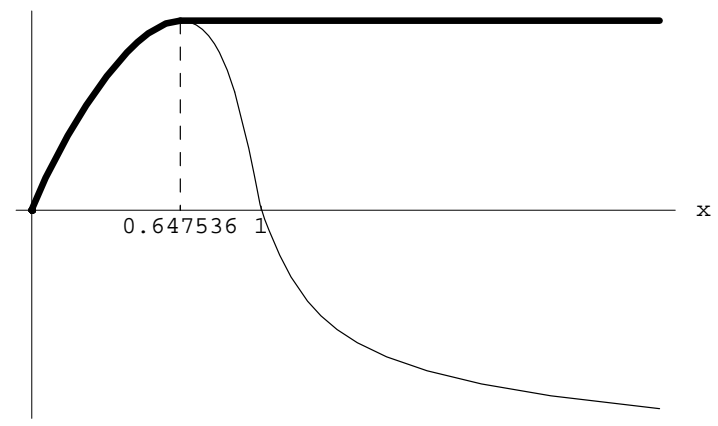

(i)

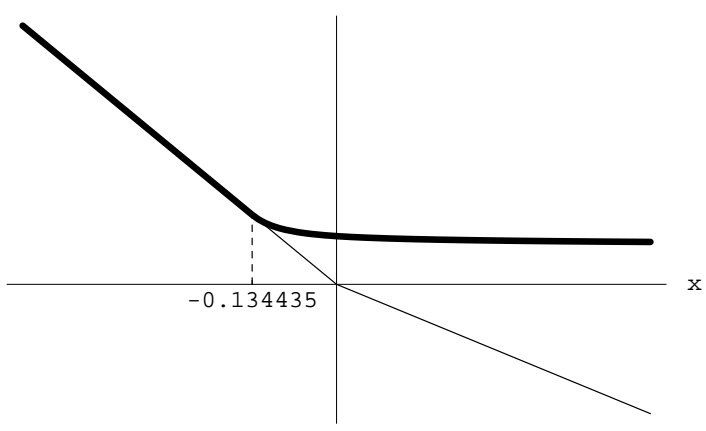

(ii)

Figure 3. $\mathbf{p}=\mathbf{- 1}$. The solid line in (i) is $H_{c}(y)$ and the thick line is $W_{c}(y)$. The solid line in (ii) is $h(x)$ and the thick line is $V_{c}(x)$. $0.647536=z_{c}$, and $-0.134435=x_{c}=$ $F^{-1}\left(z_{c}\right)$.

(ii) Symmetry : $\left(x_{1}, x_{2}\right) \in \mathcal{E}_{1}$ if and only if $\left(x_{2}, x_{1}\right) \in \mathcal{E}_{2}$. Note that this should be true even when $\sigma_{1} \neq \sigma_{2}$ (actually, Figure 4 is the case $\sigma_{1}=0.15, \sigma_{2}=0.125$ ).

(iii) Continuity : $E_{1}\left(x_{1}\right)$ and $E_{2}\left(x_{2}\right)$ are continuous at $x_{1}=0$ and $x_{2}=0$, respectively. In the region of $x_{1} \geq 0$ and $x_{2} \geq 0$, the boundaries are two straight lines (corresponding to Case (a) and (c)). The point $A:=(0.134435,0)$ and $B:=(0,0.134435)$ are obtained via $x_{c}$ and $x_{a}$, respectively. In the second and third quadrants, the curves are delineated from the analysis of Case (b). At points $A$ and $B$, the straight line and the curve look like meeting continuously.

Now we shall prove the three properties above in a rigorous manner. Note that we shall continue to use the notations $x_{a}, z_{a}, x_{b, 1}, x_{b, 2}, z_{b, 1}, z_{b, 2}, x_{c}, z_{c}$ in Section 2.3.

Proposition 3.1. $\mathcal{E}_{1}$ and $\mathcal{E}_{2}$ are convex-sets. 


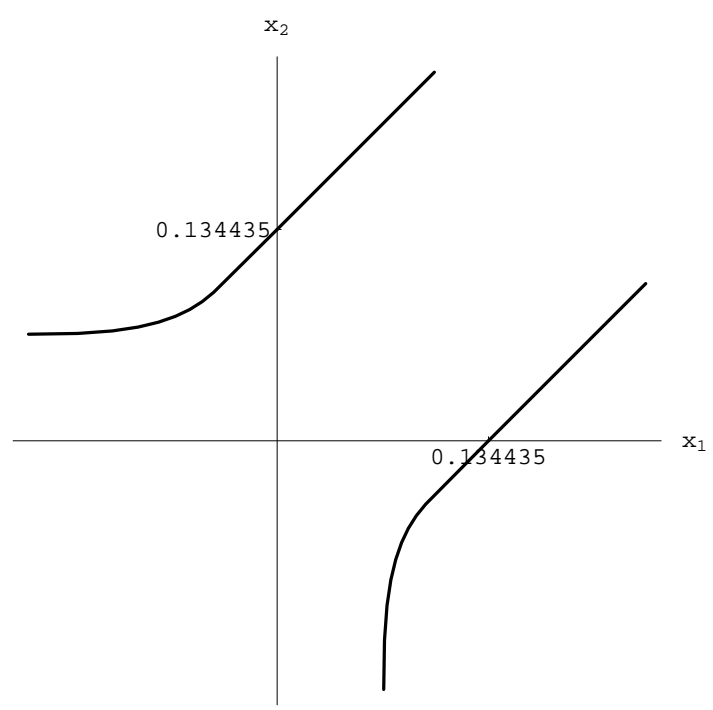

(i)

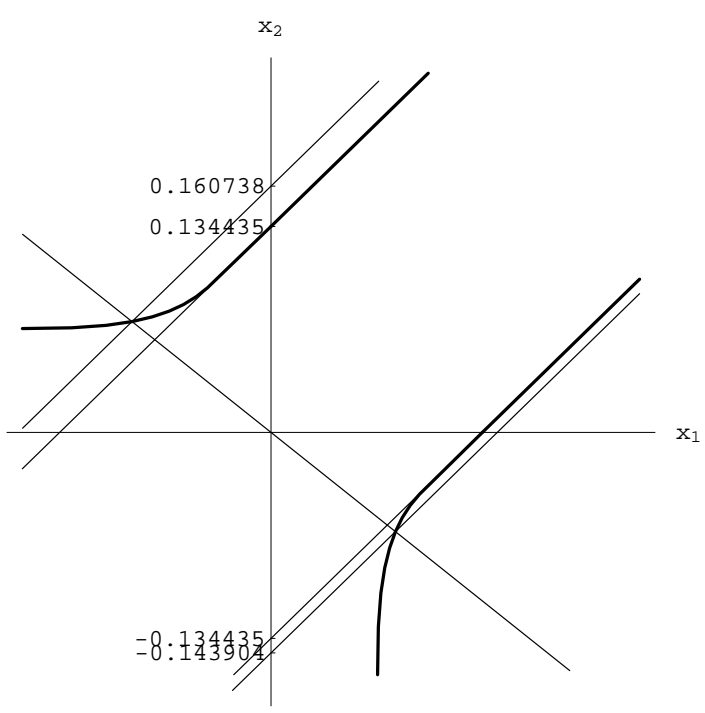

(ii)

Figure 4 . (i)The left line is $x_{2}=E_{1}\left(x_{1}\right)$, and the right line is $x_{1}=E_{2}\left(x_{2}\right)$. The exercise region $\mathcal{E}$ is $\left\{\left(x_{1}, x_{2}\right) \in \mathbb{R}^{2} \mid x_{2} \geq E_{1}\left(x_{1}\right)\right\} \cup\left\{\left(x_{1}, x_{2}\right) \in \mathbb{R}^{2} \mid x_{1} \geq E_{2}\left(x_{2}\right)\right\}$. (ii)The straight line with negative slope is $l_{p}: p x_{2}=(p-1) x_{1}$ for $p=0.55$. The line intercepting $x_{2}$-axes at $0.160738=-x_{b, 1}$ is $l_{1}: x_{1}-x_{2}=x_{b, 1}$. The line intercepting $x_{2}$-axes at $-0.143904=-x_{b, 2}$ is $l_{2}: x_{1}-x_{2}=x_{b, 2}$. Make sure " $l_{p}, l_{1}$ " and " $l_{p}, l_{2}$ " are crossed on the thick line.

Proof. We will show the convexity of $\mathcal{E}_{1}$ here. The case of $\mathcal{E}_{2}$ can be shown in the same way. The proof here is based on Broadie and Detemple [2]. First we show the convexity of the value function $V\left(x_{1}, x_{2}\right)$. Let $x=$ $\left(x_{1}, x_{2}\right) \in \mathcal{E}_{1}, x^{\prime}=\left(x_{1}^{\prime}, x_{2}^{\prime}\right) \in \mathcal{E}_{1}, \lambda \in[0,1]$ and $x(\lambda)=\left(x_{1}(\lambda), x_{2}(\lambda)\right):=\left(\lambda x_{1}+(1-\lambda) x_{1}^{\prime}, \lambda x_{2}+(1-\lambda) x_{2}^{\prime}\right)$. In addition, we define the stochastic process $N_{t}=\left(N_{t}^{1}, N_{t}^{2}\right)$, where $N_{t}^{i}:=\sigma_{i} \int_{0}^{t} e^{-\alpha(t-s)} \mathrm{d} B_{s}^{i}(i=1,2)$, then $X_{t}=\left(X_{t}^{1}, X_{t}^{2}\right)=X_{0} e^{-r t}+N_{t}$. Because the payoff function $\Phi(x)$ is convex and $N_{t}$ is independent of $X_{0}$,

$$
\begin{aligned}
V(x(\lambda)) & =\sup _{\tau \in \mathcal{S}} \mathbb{E}_{x(\lambda)}\left[e^{-r \tau} \Phi\left(\lambda\left(x e^{-\alpha \tau}+N_{\tau}\right)+(1-\lambda)\left(x^{\prime} e^{-\alpha \tau}+N_{\tau}\right)\right)\right] \\
& \leq \sup _{\tau \in \mathcal{S}} \mathbb{E}_{x(\lambda)}\left[e^{-r \tau}\left(\lambda \Phi\left(x e^{-\alpha \tau}+N_{\tau}\right)+(1-\lambda) \Phi\left(x^{\prime} e^{-\alpha \tau}+N_{\tau}\right)\right)\right] \\
& \leq \sup _{\tau \in \mathcal{S}} \mathbb{E}_{x(\lambda)}\left[e^{-r \tau} \lambda \Phi\left(x e^{-\alpha \tau}+N_{\tau}\right)\right]+\sup _{\tau \in \mathcal{S}} \mathbb{E}_{x(\lambda)}\left[e^{-r \tau}(1-\lambda) \Phi\left(x^{\prime} e^{-\alpha \tau}+N_{\tau}\right)\right] \\
& =\lambda \sup _{\tau \in \mathcal{S}} \mathbb{E}_{x}\left[e^{-r \tau} \Phi\left(x e^{-\alpha \tau}+N_{\tau}\right)\right]+(1-\lambda) \sup _{\tau \in \mathcal{S}} \mathbb{E}_{x^{\prime}}\left[e^{-r \tau} \Phi\left(x^{\prime} e^{-\alpha \tau}+N_{\tau}\right)\right] \\
& =\lambda V(x)+(1-\lambda) V\left(x^{\prime}\right) .
\end{aligned}
$$

Because $x \in \mathcal{E}_{1}$ and $x^{\prime} \in \mathcal{E}_{1}$,

$$
\begin{aligned}
V(x(\lambda)) & \leq \lambda V(x)+(1-\lambda) V\left(x^{\prime}\right) \\
& =\lambda x_{2}+(1-\lambda) x_{2}^{\prime}=x_{2}(\lambda) .
\end{aligned}
$$


On the other hand, by the definition of $V(x)$,

$$
V(x(\lambda)) \geq x_{1}(\lambda) \vee x_{2}(\lambda)=x_{2}(\lambda) .
$$

Hence it follows that $V(x(\lambda))=x_{2}(\lambda)$, which implies that $x(\lambda) \in \mathcal{E}_{1}$.

Proposition 3.2. $\left(x_{1}, x_{2}\right) \in \mathcal{E}_{1}$ if and only if $\left(x_{2}, x_{1}\right) \in \mathcal{E}_{2}$.

Proof. The lines $l_{p}$ s for $p=p^{*}$ and for $p=1-p^{*}$ are symmetric about $x_{1}=x_{2}$. Then it suffices to show the following two things:

(i) $x_{a}=-x_{c}$,

(ii) $\left\{x \in \mathbb{R} \mid V_{b}(x)>h(x)\right\}=\left(x_{b, 1}, x_{b, 2}\right)$ for $p=p^{*} \in(0,1)$ if and only if $\left\{x \in \mathbb{R} \mid V_{b}(x)>h(x)\right\}=$ $\left(-x_{b, 2},-x_{b, 1}\right)$ for $p=1-p^{*}$.

The stochastic process $-X^{e}$ satisfies the stochastic differential equation

$$
\mathrm{d}\left(-X_{t}^{e}\right)=-\alpha\left(-X_{t}^{e}\right) \mathrm{d} t+\sigma_{e} \mathrm{~d}\left(-B_{t}^{e}\right)
$$

Because $-B^{e}$ has the same distribution as $B^{e},-X^{e}$ is an Ornstein-Uhlenbeck process with the same parameters as $X^{e}$ except the sign of the initial value. In addition, the equation

$$
\begin{aligned}
h(x) & = \begin{cases}p x & (x \geq 0), \\
(p-1) x & (x<0),\end{cases} \\
& = \begin{cases}((1-p)-1)(-x) & (x \geq 0), \\
(1-p)(-x) & (x<0)\end{cases}
\end{aligned}
$$

shows that the graph of $h(x)$ with $p=p^{*}$ and that with $p=1-p^{*}$ are symmetric about $x=0$. From these observations, it is clear that (i) and (ii) hold.

Proposition 3.3. $E_{1}\left(x_{1}\right)$ and $E_{2}\left(x_{2}\right)$ are continuous at $x_{1}=0$ and $x_{2}=0$, respectively.

Proof. We show only the continuity of $E_{1}\left(x_{1}\right)$ here since that of $E_{2}\left(x_{2}\right)$ can be shown in the same way. The right hand $\operatorname{limit} \lim _{x_{1} \downarrow 0} E_{1}\left(x_{1}\right)=-x_{c}$ is clear from Proposition 2.2, and then what remains to be shown is the left hand $\operatorname{limit}_{\lim _{1} \uparrow 0} E_{1}\left(x_{1}\right)=-x_{c}$. From the procedures to draw Figure 4 and continuity of the function $F, \lim _{x_{1} \uparrow 0} E_{1}\left(x_{1}\right)=-x_{c}$ is equivalent to

$$
\lim _{p \downarrow 0} z_{b, 1}=z_{c}
$$

which we shall show in the following. For brevity, let $\zeta:=\lim _{p \downarrow 0} z_{b, 1}$ through the reminder of this proof.

(i) If $\zeta>z_{c}$, then $\lim _{p \downarrow 0} H^{\prime}\left(z_{b, 1}\right)=H^{\prime}(\zeta)<0$, since $H^{\prime}\left(z_{c}\right)=0, H^{\prime \prime}(y)<0$ for all $y \in(0,1)$, and $H^{\prime}(y)$ is continuous on $(0,1)$. Because $H^{\prime}(y)>0$ for $y \in(1, \infty)$ and $H(1)=0<H(\zeta)$, there is unique $z_{b, 2} \in(1, \infty)$ such that $H^{\prime}(\zeta)\left(z_{b, 2}-\zeta\right)+H(\zeta)=H\left(z_{b, 2}\right)$. For these $z_{b, 2}$ and $\zeta\left(\right.$ the limit of $\left.z_{b, 1}\right), W_{b}(y)$ defined in Section 3 is not concave, a contradiction to the fact $W_{b}(y)$ is the smallest concave majorant of $H(y)$. 
(ii) If $\zeta<z_{c}$, then there exists some $\zeta^{\prime} \in\left(\zeta, z_{c}\right)$, and $H^{\prime}(\zeta)>H^{\prime}\left(\zeta^{\prime}\right)>0$ because $H^{\prime}\left(z_{c}\right)=0$ and $H^{\prime \prime}(y)<0$ on $(0,1)$. We define the functions $W_{\zeta}(y)$ and $W_{\zeta^{\prime}}(y)$ by

$$
W_{\zeta}(y):= \begin{cases}H^{\prime}(\zeta)(y-\zeta) & (\zeta \leq y), \\ H(y) & (0<y<\zeta),\end{cases}
$$

and

$$
W_{\zeta^{\prime}}(y):= \begin{cases}H^{\prime}\left(\zeta^{\prime}\right)\left(y-\zeta^{\prime}\right) & \left(\zeta^{\prime} \leq y\right), \\ H(y) & \left(0<y<\zeta^{\prime}\right) .\end{cases}
$$

It is obvious from this definition that $W_{\zeta}$ and $W_{\zeta^{\prime}}$ are concave on $\mathbb{R}$. Additionally, the inequality $W_{\zeta}(y) \geq$ $W_{\zeta^{\prime}}(y) \geq H(y)$ is satisfied since $\lim _{p \downarrow 0} H(y)=0$ for $y \in(1, \infty)$. Then $W_{\zeta}(y)$ and $W_{\zeta^{\prime}}(y)$ both are concave majorants of $H(y)$ and $W_{\zeta}(y) \geq W_{\zeta^{\prime}}(y)$. This contradicts to the fact that $W_{\zeta}(y)$ is the smallest concave majorant of $H(y)$. Therefore, (3.1) must be the case and this completes the proof.

3.2. Statics about $\rho$ and $\sigma_{e}$. In this subsection, we check the effect of changing $\rho$ and $\sigma_{e}$ to exercise region $\mathcal{E}$. In this model, the change of $\rho$ only affects the value of $\sigma_{e}$, and Table 4 shows various $\rho$ and corresponding $\sigma_{e}$ with the settings of $\sigma_{1}$ and $\sigma_{2}$ not changed. Interestingly, the following observation seems to be true from the results of calculation with computer.

Observation 3.1. Let $\mathcal{E}$ be the optimal exercise region with $\sigma_{e}=\sigma^{*}$, and let $\mathcal{E}^{\prime}$ be that with $\sigma_{e}=k \sigma^{*}$ $\left(k \in \mathbb{R}_{+}\right)$. Then $\left(x_{1}, x_{2}\right) \in \mathcal{E}$ if and only if $\left(k x_{1}, k x_{2}\right) \in \mathcal{E}^{\prime}$.

In other words, if $\sigma_{e}$ changed from $\sigma^{*}$ to $k \sigma^{*}$, then the shape of exercise region doesn't change, and the scale is increased $k$ times. We shall leave a rigorous proof of this phenomenon an open problem.

\begin{tabular}{|c|c|c|c|c|}
\hline$\rho$ & -1 & 0 & 0.75 & 1 \\
\hline$\sigma_{e}$ & 0.275 & 0.195256 & 0.1 & 0.025 \\
\hline \multicolumn{5}{|c}{ TABLE 3. }
\end{tabular}

\section{Minimum Option}

We solve the problem for minimum option with the payoff function $\Psi\left(x_{1}, x_{2}\right)=x_{1} \wedge x_{2}$, while keeping the other settings the same. In the same way of reduction of the dimension in Section 2.2, we have

$$
\begin{aligned}
V\left(x_{1}, x_{2}\right) & =\sup _{\tau \in \mathcal{S}} \mathbb{E}_{x_{1}, x_{2}}\left[e^{-r \tau}\left(X_{\tau}^{1} \wedge X_{\tau}^{2}\right)\right] \\
& =\sup _{\tau \in \mathcal{S}} \mathbb{E}_{x_{1}, x_{2}}\left[-e^{-r \tau}\left(X_{\tau}^{e}\right)^{+}+e^{-r \tau} X_{\tau}^{1}\right] \\
& =\sup _{\tau \in \mathcal{S}} \mathbb{E}_{x_{1}, x_{2}}\left[e^{-r \tau}\left(-\left(X_{\tau}^{e}\right)^{+}+\frac{x_{1}}{x_{1}-x_{2}} X_{\tau}^{e}\right)\right] \\
& =\sup _{\tau \in \mathcal{S}} \mathbb{E}_{x_{1}, x_{2}}\left[e^{-r \tau} g\left(X_{\tau}^{e}\right)\right]
\end{aligned}
$$


where

$$
g(x):= \begin{cases}(1-p) x & (x \geq 0), \\ p x & (x<0) .\end{cases}
$$

The property of this function $g(x)$ is also quite different in the cases $p \geq 1,0<p<1, p \leq 0$. Because the dynamics of $X^{1}$ and $X^{2}$ are the same as before, the functions $\psi(x), \varphi(x)$, and then $F(x)$ are also the same. The function $H(y)$ is, however, different because of the change of the function from $h(x)$ to $g(x)$. The properties of this new $H(y)$ is shown in Table 3 , and in addition, we have

$$
\lim _{y \rightarrow \infty} H(y)=\left\{\begin{array}{ll}
\infty & (p>1) \\
-\infty & (p \leq 1)
\end{array}, \quad \lim _{y \rightarrow \infty} H^{\prime}(y)=0 .\right.
$$

Case (a) $p \geq 1$. In this case, the essential properties of $H(y)$ for the minimum case is the same as those for the maximum case. In fact, $z_{a}$ is also the same from Proposition 2.1 and then the smallest nonnegative concave majorant $W_{a}(y)$ of $H(y)$ becomes

$$
W_{a}(y)= \begin{cases}\frac{y H\left(z_{a}\right)}{z_{a}} & \left(0<y \leq z_{a}\right), \\ H(y) & \left(y>z_{a}\right) .\end{cases}
$$

The function $V_{a}=\varphi(x) W_{a}(F(x))$ is

$$
V_{a}(x)= \begin{cases}\frac{(p-1) x_{a}}{\psi\left(x_{a}\right)} \psi(x) & \left(x<x_{a}\right), \\ (p-1) x & \left(x \geq x_{a}\right),\end{cases}
$$

where $x_{a}=F^{-1}\left(z_{a}\right)$. Figure 5 shows the graphs of $H(y), W_{a}(y), g(x)$, and $V_{a}(x)$ with $p=2$.

Case (b) $0<p<1$. Only in this case are the properties of $H(y)$ quite different from those of the maximum option case. $H(y)$ is not positive on $\mathbb{R}_{+}$and then the smallest nonnegative concave majorant $W_{b}(y)$ of $H(y)$ is very simple: $W_{b}(y)=0$. And then $V_{b}(x)=\varphi(x) W_{b}(F(x))=0$. Figure 6 shows the graphs of $H(y), W_{b}(y), g(x)$, and $V_{b}(x)$ with $p=0.5$.

Case (c) $p \leq 0$. In this case, as in Case (a), the properties of $H(y)$ are the same as those of the maximum

\begin{tabular}{|c|c|c|c|}
\hline \multicolumn{2}{|c|}{ cases } & the sign of $H(y)$ & the sign of $H^{\prime \prime}(y)$ \\
\hline \multirow{2}{*}{$p \geq 1$} & $y \geq 1$ & + & - \\
\cline { 2 - 4 } & $0<y<1$ & - & + \\
\hline \multirow{2}{*}{$0<p<1$} & $y \geq 1$ & - & + \\
\cline { 2 - 4 } & $0<y<1$ & - & + \\
\hline \multirow{2}{*}{$p \leq 0$} & $y \geq 1$ & - & + \\
\cline { 2 - 4 } & $0<y<1$ & + & - \\
\hline
\end{tabular}

TABLE 4. 
case. $z_{c}$ is also the same from Proposition 2.2, and then the smallest nonnegative concave majorant $W_{c}(y)$ of $H(y)$ is

$$
W_{c}(y)= \begin{cases}H(y) & \left(0 \leq y<z_{c}\right) \\ H\left(z_{c}\right) & \left(y \geq z_{c}\right)\end{cases}
$$

The function $V_{c}(x)=\varphi(x) W_{c}(F(x))$ is

$$
V_{c}(x)= \begin{cases}p x & \left(x \leq x_{c}\right), \\ \frac{p x_{c}}{\varphi\left(x_{c}\right)} \varphi(x) & \left(x>x_{c}\right),\end{cases}
$$

where $x_{c}=F^{-1}\left(z_{c}\right)$. Figure 7 shows the graphs of $H(y), W_{c}(y), g(x)$, and $V_{c}(x)$ with $p=-1$.

Figure 8 shows the exercise region $\mathcal{E}$ constructed from these results. The exercise region consists of the two triangular parts. Interestingly enough, this is a subset (in fact, $\mathbb{R}_{+}^{2}$ part) of the exercise region for the maximum option (though of course, value functions are different even on this area).

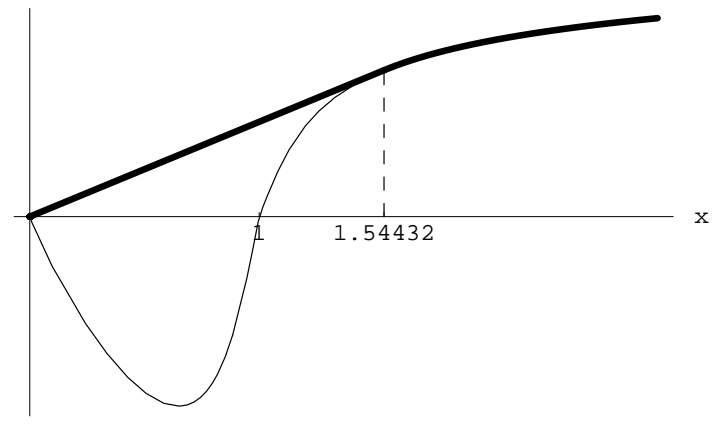

(i)

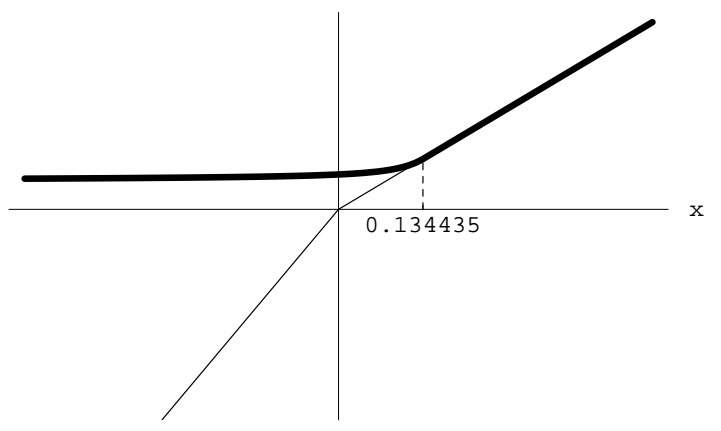

(ii)

Figure 5. $\mathbf{p}=\mathbf{2}$. The solid line in (i) is $H_{a}(y)$ and the thick line is $W_{a}(y)$. The solid line in (ii) is $h(x)$ and the thick line is $V_{a}(x) .1 .54432=z_{a}$, and $0.134435=x_{a}=F^{-1}\left(z_{a}\right)$.

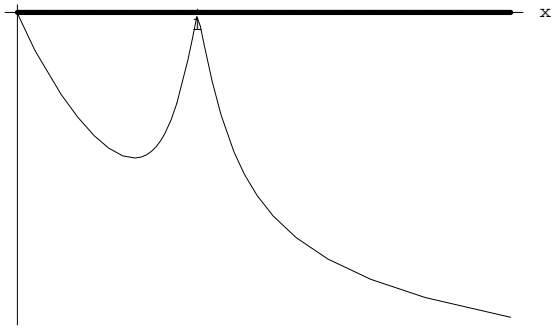

(i)

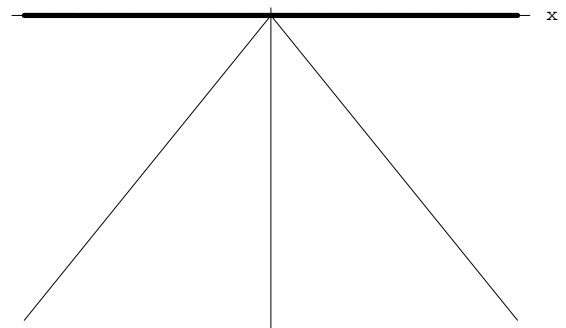

(ii)

FiguRE 6. $\mathbf{p}=\mathbf{0 . 5}$. The solid line in (i) is $H_{b}(y)$ and the thick line is $W_{b}(y)$. The solid line in (ii) is $h(x)$ and the thick line is $V_{b}(x)$. 


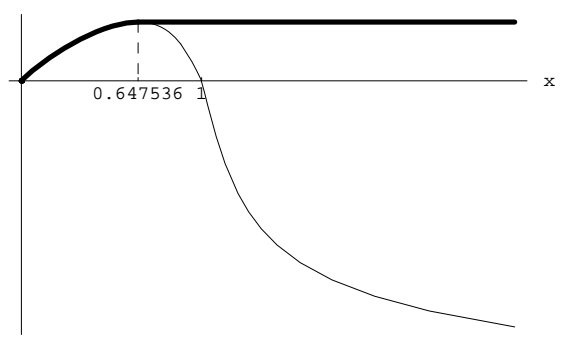

(i)

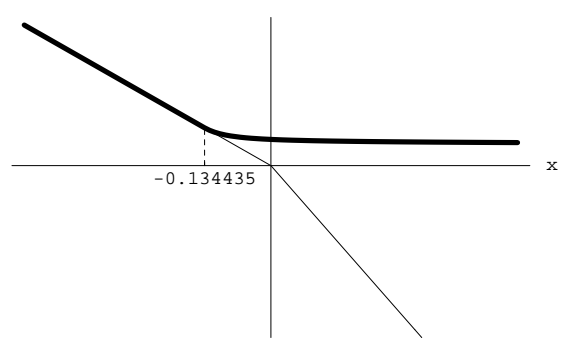

(ii)

FiguRE 7. $\mathbf{p}=\mathbf{- 1}$. The solid line in (i) is $H_{c}(y)$ and the thick line is $W_{c}(y)$. The solid line in (ii) is $h(x)$ and the thick line is $V_{c}(x) .0 .647536=z_{c}$, and $-0.134435=x_{c}=$ $F^{-1}\left(z_{c}\right)$.

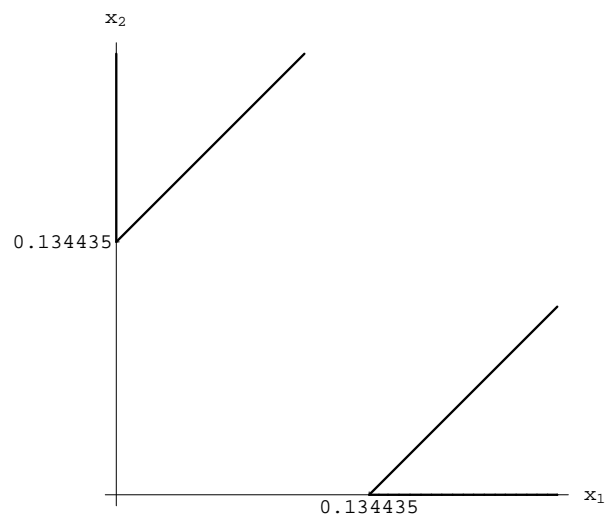

FiguRE 8. The exercise region is $\mathcal{E}=\left\{\left(x_{1}, x_{2}\right) \in \mathbb{R}^{2} \mid x_{2} \geq x_{1}+z_{a}, x_{1} \geq 0\right\} \cup\left\{\left(x_{1}, x_{2}\right) \in\right.$ $\left.\mathbb{R}^{2} \mid x_{1} \geq x_{2}+z_{a}, x_{2} \geq 0\right\}$.

\section{APPENDIX}

This appendix explains the outline of the method used in Section 2.3. The proofs and detailed explanation of the following propositions are in Dayanik and Karatzas [5]. See also Dynkin [8]. Let the stochastic process $X=\left\{X_{t} ; t \geq 0\right\}$ with state space $(a, b) \subset \mathbb{R}$ satisfy the stochastic differential equation,

$$
\mathrm{d} X_{t}=\alpha\left(X_{t}\right) \mathrm{d} t+\sigma\left(X_{t}\right) \mathrm{d} B_{t}
$$

Let the continuous function $h(x)$ be the reward function, then the value function $V(\cdot)$ is given by

$$
V(x)=\sup _{\tau \in \mathcal{S}} \mathbb{E}_{x}\left[e^{-\beta \tau} h\left(X_{\tau}\right)\right] .
$$

We shall define the differential operator $\mathcal{A}$ by

$$
\mathcal{A} u(\cdot)=\frac{1}{2} \sigma^{2}(\cdot) \frac{\mathrm{d}^{2} u}{\mathrm{~d} x^{2}}(\cdot)+\alpha(\cdot) \frac{\mathrm{d} u}{\mathrm{~d} x}(\cdot), \quad u \in \mathcal{C}^{2}
$$


The increasing and decreasing solutions $\psi(\cdot)$ and $\varphi(\cdot)$ of ordinary differential equation $(\mathcal{A}-\beta) u(\cdot)=0$ are denoted by

$$
\begin{aligned}
& \psi(x)= \begin{cases}\mathbb{E}_{x}\left[e^{-\beta \tau_{c}} 1_{\tau_{c}<\infty}\right] & (x \leq c) \\
1 / \mathbb{E}_{c}\left[e^{-\beta \tau_{x}} 1_{\tau_{x}<\infty}\right] & (x>c)\end{cases} \\
& \varphi(x)= \begin{cases}1 / \mathbb{E}_{c}\left[e^{-\beta \tau_{x}} 1_{\tau_{x}<\infty}\right] & (x \leq c) \\
\mathbb{E}_{x}\left[e^{-\beta \tau_{c}} 1_{\tau_{c}<\infty}\right] & (x>c)\end{cases}
\end{aligned}
$$

for arbitrary fixed $c \in(a, b)$, where $\tau_{z}=\inf \left\{t \geq 0 ; X_{t}=z\right\}$ for $z \in(a, b)$. A function $u$ is called $F$-concave if, for every $a \leq l<r \leq b$ and $x \in[l, r]$, we have

$$
u(x) \geq u(l) \frac{F(r)-F(x)}{F(r)-F(l)}+u(r) \frac{F(x)-F(l)}{F(r)-F(l)} .
$$

Proposition 5.1. $U(x) / \varphi(x)$ is $F$-concave if and only if $U(x)$ is $\beta$-excessive, i.e,

$$
U(x) \geq \mathbb{E}\left[e^{-\beta \tau} U\left(X_{\tau}\right)\right], \forall \tau \in \mathcal{S}, \forall x \in(a, b) .
$$

Proposition 5.2. $V(x)$ is the smallest nonnegative majorant of $h(x)$ such that $V(x) / \varphi(x)$ is $F$-concave.

We shall define the value $l_{a}$ and $l_{b}$ by

$$
l_{a}:=\limsup _{x \downarrow a}\left(h^{+} / \varphi\right)(x), \quad l_{b}:=\limsup _{x \uparrow b}\left(h^{+} / \psi\right)(x) .
$$

Proposition 5.3. Let $W(y):[0, \infty) \rightarrow \mathbb{R}$ be the smallest nonnegative concave majorant of

$$
H(y):=\left\{\begin{array}{ll}
(h / \varphi) \circ F^{-1}(y) & (y>0) \\
l_{a} & (y=0)
\end{array},\right.
$$

then the value function $V$ is denoted by $V(x)=\varphi(x) W(F(x))$.

Proposition 5.4. The value function $V(x)$ is continuous. If $l_{a}=l_{b}=0$, then the optimal stopping rule of $(5,1)$ is

$$
\tau^{*}=\inf \left\{t \geq 0 ; X_{t} \in \mathcal{E}\right\}
$$

where the exercise region $\mathcal{E}$ is given by

$$
\mathcal{E}=\{x \in[c, d]: V(x)=h(x)\}
$$

\section{REFERENCES}

[1] E. Bayraktar and M. Egami. On the one-dimensional optimal switching problem. Mathematics of Operations Research, 35 (1):140-159, 2010.

[2] M. Broadie and J. Detemple. The valuation of American options on multiple assets. Mathematical Finance, 7:241-286, 1997.

[3] A. Cadenillas, S. Sarkar, and F. Zapatero. Optimal dividend policy with mean-reverting cash reservoir. Mathematical Finance, 17 (1):81-109, 2007. 
[4] M. H. A. Davis and A. R. Norman. Portfolio selection with transaction costs. Mathematics of Operations Research, 15:676-713, 1990.

[5] S. Dayanik and I. Karatzas. On the optimal stopping problem for one-dimensional diffusions. Stochastic Processes and their Applications, 107 (2):173-212, 2003.

[6] J. Detemple. American-Style Derivatives: Valuation and Computation. Chapman and Hall/CRC, London, 2005.

[7] J. Detemple, S. Feng, and W. Tian. The valuation of American call options on the minimum of two dividend-paying assets. Annals of Applied Probability, 13:953-983, 2003.

[8] E. Dynkin. Markov processes, Volume II. Springer Verlag, Berlin, 1965.

[9] T. Guillaume. Making the best of best-of. Review of Derivative Research, 11:1-39, 2008.

[10] N. N. Levedev. Special Functions and Their Applications. Dover Publications, New York, 1972.

[11] S. Villeneuve. Excercise regions of American options on several assets. Finance and Stochastics, 3:295-322, 1999.

(M. Egami) Graduate School of Economics, Kyoto University, Sakyo-Ku, Kyoto, 606-8501, Japan E-mail address: egami@econ.kyoto-u.ac.jp

(T. Oryu) Graduate School of Economics, Kyoto University, Sakyo-Ku, Kyoto, 606-8501, Japan E-mail address: oryu@eco06.mbox.media.kyoto-u.ac.jp 